\title{
Diagnostic checking for Non-stationary ARMA Models: An Application to Financial Data
}

\author{
S.-Q. Ling ${ }^{\mathrm{a}}$, K. Zhu ${ }^{\mathrm{b}}$, C.-Y. Chong ${ }^{\mathrm{a}}$ \\ ${ }^{\mathrm{a}}$ Hong Kong University of Science and Technology \\ ${ }^{\mathrm{b}}$ Chinese Academy of Sciences \\ Email: maling@ust.hk
}

\begin{abstract}
This paper first derives the limiting distributions of the residual and the squared residual autocorrelation functions of the nonstationary autoregressive moving-average model, respectively. We then use them to construct two portmanteau statistics for testing the adequacy of the fitted model. Simulation results show that the tests have reasonable empirical sizes and powers in the finite samples.
\end{abstract}

Keywords: Portmanteau test, Nonstationary ARMA, Residual ACFs, Squared residual ACFs 


\section{INTRODUCTION}

Diagnostic checking is one of the most important steps in time series modeling. Box and Pierce (1970) proposed a portmanteau statistic based on the residual autocorrelation functions (ACFs) of the autoregressive moving-average (ARMA) model for testing the adequacy of the fitted model. Ljung and Box (1978) suggested a simple test statistic, which is a modification of Box and Pierce's statistic, and is easy to calculate. McLeod (1978) extended their results directly to more general situations. McLeod and Li (1983) proposed a new portmanteau statistic based on the squared residual ACFs. All these tests were developed for stationary time series models.

Shin and Lee (1996) proposed a portmanteau statistic for the nonstationary AR model. This statistic is asymptotically follows a $\chi^{2}$ distribution. However, the corresponding result has not been established for the nonstationary ARMA (NARMA) model. Testing the unit root in the NARMA model is one of the most important issues in studies of times series and econometrics. The Dickey-Fuller tests have been extensively used in the literature. However, an adequate model is essential for the use of these tests. This paper first derives the limiting distributions of the residual ACFs and the squared residual ACFs of the NARMA model, respectively, and then uses them to construct two portmanteau statistics.

Throughout this paper, the following notation are commonly used. $O(1)\left(O_{p}(1)\right)$ denotes a series of numbers (random numbers) that are bounded (in probability). $o(1)\left(o_{p}(1)\right)$ denotes a series of numbers (random numbers) converging to zero (in probability). $\stackrel{D}{\longrightarrow}$ and $\stackrel{p}{\longrightarrow}$ denote convergence in distribution and in probability, respectively. $V^{\prime}$ denotes the transpose of vector $V . D[0,1]$ denotes the space of function $f(s)$ on $[0,1]$, which is defined and equipped with the Skorokhod topology in Billingsley (1968).

\section{REVIEW OF NONSTATIONARY ARMA MODELS}

In this section, we review some results on the estimation of the NARMA model.

\subsection{MODELS}

Let $\left\{Y_{t}\right\}_{t=1}^{n}$ be a set of observations generated by the $\operatorname{NARMA}(p, q)$ model,

$$
\phi(L) Y_{t}=\Theta(L) \varepsilon_{t},
$$

where $\left\{\varepsilon_{t}\right\}$ is a sequence of unobservable independent and identically distributed (i.i.d.) errors with mean zero and unknown variance $\sigma_{\varepsilon}^{2}, \phi(L)=1-\phi_{1} L-\cdots-\phi_{p} L^{p}$ and $\theta(L)=1-\theta_{1} L-\cdots-\theta_{q} L^{q}$. We assume that $\phi(L)=0$ has one root that is equal to 1 and its other roots are outside the unit circle, and all the roots of $\theta(L)=0$ lie outside the unit circle. Model (1) is nonstationary in this case.

Let $W_{t}=Y_{t}-Y_{t-1}$. Then, model (1) can be expressed in the error-correction form as

$$
W_{t}=C Y_{t-1}+\sum_{j=1}^{p-1} \phi_{j}^{*} W_{t-j}+\varepsilon_{t}-\sum_{j=1}^{q} \theta_{j} \varepsilon_{t-j}
$$

where $C=-\phi(1)=-\left(1-\sum_{j=1}^{p} \phi_{j}\right)$ and $\phi_{j}^{*}=-\sum_{i=j+1}^{p} \phi_{i}$. We let $\phi^{*}(L)=1-\sum_{j=1}^{p-1} \phi_{j}^{*} L^{j}$. Since $C=-\phi(1)=-\left(1-\sum_{j=1}^{p} \phi_{j}\right)=0, W_{t}$ has a stationary representation,

$$
W_{t}=\psi_{\theta}(L) \varepsilon_{t}
$$

where $\psi_{\theta}(L)=\psi(L) \theta(L)$ and $\psi(L)=\phi^{*-1}(L)=\left(1-\sum_{j=1}^{p-1} \phi_{j}^{*} L^{j}\right)^{-1}=\sum_{k=0}^{\infty} \psi_{k} L^{k}, \psi_{0}=1$, and $\psi_{k}=O\left(\rho^{k}\right)$ with $\rho \in(0,1)$, see Hannan (1970, p.11).

\subsection{LIMITING DISTRIBUTION OF GAUSSIAN ESTIMATORS}

Denote $\beta=\left[C, \alpha^{\prime}\right]^{\prime}$ and $\beta_{0}$ be its true value, where $\alpha=\left[\phi^{* \prime}, \theta^{\prime}\right]^{\prime}, \phi^{*}=\left[\phi_{1}^{*}, \cdots, \phi_{p-1}^{*}\right]^{\prime}$ and $\theta=\left[\theta_{1}, \cdots, \theta_{q}\right]^{\prime}$. Let $\hat{\beta}$ be the Gaussian estimator of $\beta$. Taking the first-order partial derivatives with respect to $\beta$ in (2), we have

$$
X_{t-1} \equiv-\frac{\partial \varepsilon_{t}}{\partial \beta}=\left[-\frac{\partial \varepsilon_{t}}{\partial C}, U_{t-1}^{\prime}\right]^{\prime}
$$


S.-Q. Ling, K. Zhu and C.-Y. Chong, Diagnostic checking for Non-stationary ARMA Models

where $U_{t-1}=-\partial \varepsilon_{t} / \partial \alpha . X_{t}$ satisfies the following recursive equation:

$$
\left(1-\sum_{j=1}^{q} \theta_{j} L^{j}\right) X_{t-1}=\left[Y_{t-1}, W_{t-1}, \cdots, W_{t-p+1},-\varepsilon_{t-1}, \cdots,-\varepsilon_{t-q}\right]^{\prime} \equiv X_{t-1}^{*} .
$$

Similar to (4), the vector $U_{t}$ satisfies the following recursive equation:

$$
\left(1-\sum_{j=1}^{q} \theta_{j} L^{j}\right) U_{t-1}=\left[W_{t-1}, \cdots, W_{t-p+1},-\varepsilon_{t-1}, \cdots,-\varepsilon_{t-q}\right]^{\prime} \equiv U_{t-1}^{*} .
$$

Note that $U_{t}^{*}$ is stationary. The following Lemma 2.1 is Proposition 17.3 in Hamilton (1994).

Lemma 2.1 Let $W_{t}=\sum_{j=0}^{\infty} \widetilde{\psi}_{j} \varepsilon_{t-j}$, where $\widetilde{\psi}_{j}=O\left(\rho^{j}\right)$ with $\rho \in(0,1)$. Define $\gamma_{j}=E\left(W_{t} W_{t-j}\right)$ $=\sigma_{\varepsilon}^{2} \sum_{s=0}^{\infty} \widetilde{\psi}_{s} \widetilde{\psi}_{s+j}$ for $j=1,2, \cdots, \lambda=\sigma_{\varepsilon} \sum_{j=0}^{\infty} \widetilde{\psi}_{j}$ and $Y_{t}=\sum_{s=1}^{t} W_{s}$ for $t=1, \cdots, n$ with $Y_{0}=$ 0 . Then

(i) $\frac{1}{\sqrt{n}} \sum_{t=1}^{n} W_{t} \stackrel{D}{\longrightarrow} \lambda \omega(\tau)$ in $D[0,1]$,

(ii) $\frac{1}{\sqrt{n}} \sum_{t=1}^{n} W_{t-j} \varepsilon_{t} \stackrel{D}{\longrightarrow} N\left(0, \sigma_{\varepsilon}^{2} \gamma_{0}\right)$ for $j=1,2, \cdots$,

(iii) $\frac{1}{n} \sum_{t=1}^{n} Y_{t-1} \varepsilon_{t} \stackrel{D}{\longrightarrow} \sigma_{\varepsilon} \lambda \int_{0}^{1} \omega(\tau) d \omega(\tau)$,

(iv) $\frac{1}{n} \sum_{t=1}^{n} Y_{t-1} W_{t-j} \stackrel{D}{\longrightarrow} \begin{cases}\frac{1}{2}\left\{\lambda^{2} \omega^{2}(1)-\gamma_{0}\right\} & \text { for } j=0 . \\ \frac{1}{2}\left\{\lambda^{2} \omega^{2}(1)-\gamma_{0}\right\}+\sum_{i=0}^{j-1} \gamma_{i} & \text { for } j=1,2, \cdots\end{cases}$

The following Theorem 2.1 is Theorem 1 in Yap and Reinsel (1995).

Theorem 2.1 Under the assumptions of model (1), it follows that

$$
\text { (i) } \begin{aligned}
n(\hat{C}-C)= & \Theta\left(\frac{1}{n} \sum_{t=1}^{n} Y_{t-1} \varepsilon_{t}\right)\left(\frac{1}{n^{2}} \sum_{t=1}^{n} Y_{t-1}^{2}\right)^{-1}+o_{p}(1) \\
& \stackrel{D}{\longrightarrow}\left\{\int_{0}^{1} B(u) d B(u)\right\}\left\{\int_{0}^{1} B^{2}(u) d u\right\}^{-1} \Psi^{-1}
\end{aligned}
$$

where $\Theta=\theta(1)=1-\sum_{j=1}^{q} \theta_{j}, \Psi=\psi(1)=\phi^{*-1}(1)$ and $B(u)$ is a standard Brownian motion;

$$
\text { (ii) } \begin{aligned}
\sqrt{n}\left(\hat{\alpha}-\alpha_{0}\right) & =\left(\frac{1}{n} \sum_{t=1}^{n} \frac{U_{t-1} U_{t-1}^{\prime}}{\sigma_{\varepsilon}^{2}}\right)^{-1}\left(\frac{1}{\sqrt{n}} \sum_{t=1}^{n} \frac{U_{t-1} \varepsilon_{t}}{\sigma_{\varepsilon}^{2}}\right)+o_{p}(1) \\
& \stackrel{D}{\longrightarrow} N\left(0, V^{-1}\right)
\end{aligned}
$$

where $V=E\left(U_{t-1} U_{t-1}^{\prime}\right) / \sigma_{\varepsilon}^{2}$.

\section{MAIN RESULTS}

This section discusses the main results of the paper. We first derive the limiting distributions of the residual ACFs and the squared residual ACFs of model (1) and then use them to construct the portmanteau statistics.

\subsection{LIMITING DISTRIBUTION OF THE RESIDUAL ACFS}

Let $\varepsilon_{t}(\beta)$ be defined as

$$
\varepsilon_{t}(\beta)=\sum_{j=1}^{q} \theta_{j} \varepsilon_{t-j}(\beta)+W_{t}-C Y_{t-1}-\sum_{j=1}^{p-1} \phi_{j}^{*} W_{t-j}
$$

Denote $\hat{\varepsilon}_{t}=\varepsilon_{t}(\hat{\beta})$, where $\hat{\beta}$ is defined as in Section 2. Thus, $\hat{\varepsilon}_{t}$ is the residual of model (1) when $\beta_{0}$ is estimated by $\hat{\beta}$. 
The residual autocovariance $(\mathrm{ACV})$ is defined as

$$
\hat{\gamma}_{k}=\frac{1}{n} \sum_{t=1}^{n-k} \hat{\varepsilon}_{t} \hat{\varepsilon}_{t+k}
$$

When $k=0$, we can show that

$$
\hat{\gamma}_{0}=\frac{1}{n} \sum_{t=1}^{n} \hat{\varepsilon}_{t}^{2} \stackrel{p}{\longrightarrow} \sigma_{\varepsilon}^{2}
$$

as $n \longrightarrow \infty$. The corresponding residual ACF is defined as

$$
\hat{\rho}_{k}=\frac{\hat{\gamma}_{k}}{\hat{\gamma}_{0}}=\frac{\sum_{t=1}^{n-k} \hat{\varepsilon}_{t} \hat{\varepsilon}_{t+k}}{\sum_{t=1}^{n} \hat{\varepsilon}_{t}^{2}}
$$

Denote $\hat{\rho}=\left(\hat{\rho}_{1}, \cdots, \hat{\rho}_{M}\right)^{\prime}$. The aim of this section is to derive the limiting distribution of the vector $\sqrt{n} \hat{\rho}$. We first consider $\hat{\gamma}_{k}$. (8) can be rewritten as

$$
\varepsilon_{t}(\beta)=W_{t}-\beta^{\prime} X_{t-1}^{*}
$$

where $X_{t-1}^{*}=\left[Y_{t-1}, \widetilde{W}_{t-1}^{\prime},-\widetilde{\varepsilon}_{t-1}(\beta)^{\prime}\right]^{\prime}, \widetilde{W}_{t-1}=\left[W_{t-1}, \cdots, W_{t-p+1}\right]^{\prime}$ and $\widetilde{\varepsilon}_{t-1}(\beta)=\left[\varepsilon_{t-1}(\beta), \cdots\right.$, $\left.\varepsilon_{t-q}(\beta)\right]^{\prime}$. Since $\varepsilon_{t}\left(\beta_{0}\right)=\varepsilon_{t}$, by Taylor's expansion, we have

$$
\begin{aligned}
\frac{1}{\sqrt{n}} \sum_{t=1}^{n-k} \hat{\varepsilon}_{t} \hat{\varepsilon}_{t+k}= & \frac{1}{\sqrt{n}} \sum_{t=1}^{n-k} \varepsilon_{t}(\hat{\beta}) \varepsilon_{t+k}(\hat{\beta}) \\
= & \frac{1}{\sqrt{n}} \sum_{t=1}^{n-k} \varepsilon_{t} \varepsilon_{t+k}+\left.\frac{1}{\sqrt{n}} \sum_{t=1}^{n-k}\left(\hat{\beta}-\beta_{0}\right)^{\prime} \frac{\partial\left[\varepsilon_{t}(\beta) \varepsilon_{t+k}(\beta)\right]}{\partial \beta}\right|_{\beta=\beta_{0}} \\
& +\left.\frac{1}{2 \sqrt{n}} \sum_{t=1}^{n-k}\left(\hat{\beta}-\beta_{0}\right)^{\prime} \frac{\partial^{2}\left[\varepsilon_{t}(\beta) \varepsilon_{t+k}(\beta)\right]}{\partial \beta \partial \beta^{\prime}}\right|_{\beta=\beta^{*}}\left(\hat{\beta}-\beta_{0}\right) \\
\equiv & A_{1}+A_{2}+A_{3}
\end{aligned}
$$

where $\beta^{*}=\beta_{0}+\rho\left(\hat{\beta}-\beta_{0}\right)$ with $|\rho|<1$. Then, we give two lemmas and their proofs are given in the Appendix.

Lemma 3.1 Under the assumption of model (1), it follows that

(i) $\frac{1}{n \sqrt{n}} \sum_{t=1}^{n-k}\left[Y_{t+k-h-1} \varepsilon_{t}\right]=o_{p}(1)$,

(ii) $\frac{1}{n \sqrt{n}} \sum_{t=1}^{n-k}\left[Y_{t-h-1} \varepsilon_{t+k}\right]=o_{p}(1)$,

(iii) $\frac{1}{n} \sum_{t=1}^{n-k}\left[\theta^{-1}(L) \widetilde{W}_{t+k-1}\right] \varepsilon_{t} \stackrel{p}{\longrightarrow} E\left[\theta^{-1}(L) \widetilde{W}_{t+k-1} \varepsilon_{t}\right]$,

(iv) $\frac{1}{n} \sum_{t=1}^{n-k}\left[\theta^{-1}(L) \widetilde{W}_{t-1}\right] \varepsilon_{t+k}=o_{p}(1)$,

(v) $\frac{1}{n} \sum_{t=1}^{n-k}\left[\theta^{-1}(L) \widetilde{\varepsilon}_{t+k-1}\right] \varepsilon_{t} \stackrel{p}{\longrightarrow} E\left[\theta^{-1}(L) \widetilde{\varepsilon}_{t+k-1} \varepsilon_{t}\right]$,

(vi) $\frac{1}{n} \sum_{t=1}^{n-k}\left[\theta^{-1}(L) \widetilde{\varepsilon}_{t-1}\right] \varepsilon_{t+k}=o_{p}(1)$.

Lemma 3.2 Under the assumption of model (1),

(i) $A_{2}=-\left\{E\left(\frac{U_{t-1} U_{t-1}^{\prime}}{\sigma_{\varepsilon}^{2}}\right)^{-1}\left(\frac{1}{\sqrt{n}} \sum_{t=1}^{n-k} \frac{U_{t+k-1} \varepsilon_{t+k}}{\sigma_{\varepsilon}^{2}}\right)\right\}^{\prime} E\left(U_{t+k-1} \varepsilon_{t}\right)+o_{p}(1)$,

(ii) $A_{3}=o_{p}(1)$.

Thus, we have the following theorem. 
Theorem 3.1 Under the assumption of model (1) or (2),

$$
\sqrt{n} \hat{\rho}=\sqrt{n}\left(\hat{\rho}_{1}, \cdots, \hat{\rho}_{M}\right)^{\prime} \stackrel{D}{\longrightarrow} N(0, \Sigma)
$$

where $\Sigma=I_{M}-D^{\prime} \Omega^{-1} D, \Omega^{-1}=E\left(U_{t-1} U_{t-1}^{\prime}\right)^{-1}, D_{k}=E\left(U_{t+k-1} \varepsilon_{t}\right)$ and $D=\left(D_{1}, \cdots, D_{M}\right) / \sigma_{\varepsilon}$.

We consider the hypothesis:

$$
\begin{aligned}
& H_{0}: \operatorname{Model}(2) \quad \text { is correct vs } \\
& H_{1}: \operatorname{Model}(2) \quad \text { is not correct }
\end{aligned}
$$

The portmanteau statistic is defined as

$$
Q_{M}=n \hat{\rho}^{\prime}\left(I_{M}-D^{\prime} \Omega^{-1} D\right)^{-1} \hat{\rho} .
$$

By Theorem 3.1, under $H_{0}, Q_{M}$ is asymptotically follows a $\chi^{2}$ distribution with $M$ degrees of freedom. $Q_{M}$ can be used to test the hypothesis $H_{0}$ against $H_{1}$. When $H_{0}$ is accepted, we can claim that model (2) is adequate for the data $\left\{Y_{t}\right\}_{t=1}^{n}$.

\subsection{LINITING DISTRIBUTION OF THE SQUARED RESIDUAL ACFS}

We define the squared residual autocovariance $(\mathrm{ACV})$ as

$$
\hat{\gamma}_{k}=\frac{1}{n} \sum_{t=1}^{n-k}\left(\hat{\varepsilon}_{t}^{2}-1\right)\left(\hat{\varepsilon}_{t+k}^{2}-1\right) .
$$

We assume $E \varepsilon_{t}^{2}=\sigma_{\varepsilon}^{2}=1$. When $\sigma_{\varepsilon}^{2} \neq 1, \sigma_{\varepsilon}^{2}$ can be estimated by $\hat{\sigma}_{\varepsilon}^{2}$ defined in (10). When $k=0$, we can show that

$$
\hat{\gamma}_{0}=\frac{1}{n} \sum_{t=1}^{n}\left(\hat{\varepsilon}_{t}^{2}-1\right)^{2} \stackrel{p}{\longrightarrow} E \varepsilon_{t}^{4}-1 \equiv \sigma_{4},
$$

as $n \longrightarrow \infty$. The corresponding squared residual ACF is defined as

$$
\tilde{r}_{k}=\frac{\hat{\gamma}_{k}}{\hat{\gamma}_{0}}=\frac{\sum_{t=1}^{n-k}\left(\hat{\varepsilon}_{t}^{2}-1\right)\left(\hat{\varepsilon}_{t+k}^{2}-1\right)}{\sum_{t=1}^{n}\left(\hat{\varepsilon}_{t}^{2}-1\right)^{2}} .
$$

Denote $\widetilde{R}=\left(\tilde{r}_{1}, \cdots, \tilde{r}_{M}\right)^{\prime}$. The aim of this section is to derive the limiting distribution of the vector $\sqrt{n} \widetilde{R}$. Now, we first consider $\hat{\gamma}_{k}$. Since $\varepsilon_{t}^{2}\left(\beta_{0}\right)=\varepsilon_{t}^{2}$, by Taylor's expansion, we have

$$
\begin{aligned}
\frac{1}{\sqrt{n}} \sum_{t=1}^{n-k}\left(\hat{\varepsilon}_{t}^{2}-1\right)\left(\hat{\varepsilon}_{t+k}^{2}-1\right) \\
=\frac{1}{\sqrt{n}} \sum_{t=1}^{n-k}\left(\varepsilon_{t}^{2}(\hat{\beta})-1\right)\left(\varepsilon_{t+k}^{2}(\hat{\beta})-1\right) \\
=\frac{1}{\sqrt{n}} \sum_{t=1}^{n-k}\left(\varepsilon_{t}^{2}-1\right)\left(\varepsilon_{t+k}^{2}-1\right) \\
\quad+\left.\frac{1}{\sqrt{n}} \sum_{t=1}^{n-k}\left(\hat{\beta}-\beta_{0}\right)^{\prime} \frac{\partial\left[\left(\varepsilon_{t}^{2}(\beta)-1\right)\left(\varepsilon_{t+k}^{2}(\beta)-1\right)\right]}{\partial \beta}\right|_{\beta=\beta_{0}},\left.\frac{\partial^{2}\left[\left(\varepsilon_{t}^{2}(\beta)-1\right)\left(\varepsilon_{t+k}^{2}(\beta)-1\right)\right]}{\partial \beta \partial \beta^{\prime}}\right|_{\beta=\beta^{*}}\left(\hat{\beta}-\beta_{0}\right) \\
\quad+\frac{1}{2 \sqrt{n}} \sum_{t=1}^{n-k}\left(\hat{\beta}-\beta_{0}\right) \frac{B_{1}}{\equiv}
\end{aligned}
$$


where $\beta^{*}=\beta_{0}+\rho\left(\hat{\beta}-\beta_{0}\right)$, with $|\rho|<1$. Then, we give two basic lemmas and their proofs are given in the Appendix.

Lemma 3.3 Suppose $Y_{t}, \widetilde{W}_{t-1}$, and $\widetilde{\varepsilon}_{t-1}$ are defined as in (12). Let $\xi_{t}=\varepsilon_{t}\left(\varepsilon_{t+k}^{2}-1\right)$ and $\xi_{t+k}=$ $\varepsilon_{t+k}\left(\varepsilon_{t}^{2}-1\right)$. If $\sigma_{4}<\infty$, then it follows that

(i) $\frac{1}{n \sqrt{n}} \sum_{t=1}^{n-k}\left[Y_{t+k-h-1} \xi_{t+k}\right]=o_{p}(1)$,

(ii) $\frac{1}{n \sqrt{n}} \sum_{t=1}^{n-k}\left[Y_{t-h-1} \xi_{t}\right]=o_{p}(1)$,

(iii) $\frac{1}{n} \sum_{t=1}^{n-k}\left[\theta^{-1}(L) \widetilde{W}_{t+k-1}\right] \xi_{t+k}=o_{p}(1)$,

(iv) $\frac{1}{n} \sum_{t=1}^{n-k}\left[\theta^{-1}(L) \widetilde{W}_{t-1}\right] \xi_{t}=o_{p}(1)$,

(v) $\frac{1}{n} \sum_{t=1}^{n-k}\left[\theta^{-1}(L) \widetilde{\varepsilon}_{t+k-1}\right] \xi_{t+k}=o_{p}(1)$,

(vi) $\frac{1}{n} \sum_{t=1}^{n-k}\left[\theta^{-1}(L) \widetilde{\varepsilon}_{t-1}\right] \xi_{t}=o_{p}(1)$.

Lemma 3.4 Under the assumption of model (1),

(i) $B_{2}=o_{p}(1)$,

(ii) $B_{3}=o_{p}(1)$.

Thus, we have the following theorem.

Theorem 3.2 If the assumption of model (1) or (2) holds and $\sigma_{4}<\infty$, then

$$
\sqrt{n} \widetilde{R}=\sqrt{n}\left(\tilde{r}_{1}, \cdots, \tilde{r}_{M}\right)^{\prime} \stackrel{D}{\longrightarrow} N\left(0, I_{M}\right) .
$$

We consider the following hypothesis:

$$
\begin{array}{ll}
H_{0}: & \operatorname{Model}(2) \quad \text { is correct vs } \\
H_{1}: & \operatorname{Model}(2) \quad \text { is not correct }
\end{array}
$$

The new portmantaue statistic is defined as

$$
\widetilde{Q}_{M}=n \widetilde{R}^{\prime} \widetilde{R} .
$$

By Theorem 3.2, under $H_{0}, \widetilde{Q}_{M}$ is asymptotically follows a $\chi^{2}$ distribution with $M$ degrees of freedom. $\widetilde{Q}_{M}$ can be used to test the hypothesis $H_{0}$ against $H_{1}$. When $H_{0}$ is accepted, we can claim that model (2) is adequate for the data $\left\{Y_{t}\right\}_{t=1}^{n}$.

ACKNOWLEDGEMENT The authors greatly appreciates the helpful comments and suggestions from the reviewer and Professor M. McAleer. This work is supported in part by Hong Kong Research Grants Commission Grants (Grant HKUST641912 and FSGRF12SC12) and NSFC (No. 11201459).

\section{REFERENCES}

Billingsley, P. (1968) Convergence of Probability Measures. New York: Wiley.

Box, G.E.P. and Pierce, D.A. (1970) Distribution of the residual autocorrelations in autoregressive integrated moving average time series models. Journal of the American Statistical Association 65, 1509-1526.

Hamilton, J.D. (1994) Time Series Analysis. United Kingdom: Princeton University Press.

Hannan, E.J. (1970) Multiple Time Series. New York: Wiley.

Ljung, G.M. and Box, G.E.P. (1978) On a measure of lack of fit in time series models. Biometrika $\mathbf{6 5}$, 297-303.

McLeod, A.I. (1978) On the distribution of residual autocorrelations in Box-Jenkins model. Journal of the Royal Statistical Society, Series B 40, 296-302. 
S.-Q. Ling, K. Zhu and C.-Y. Chong, Diagnostic checking for Non-stationary ARMA Models

McLeod, A.I. and LI, W.K. (1983) Disgnostic checking ARMA times series models using squared-residual autocorrelations. Journal of Time Series Analysis 4, 269-273.

Shin, D.W. and Lee, J.H. (1996) Distribution of residual autocorrelations in nonstationary autoregressive processes. Journal of Time Series Analysis 17, 105-109.

Yap, S.F. and Reinsel, G.C. (1995) Results on estimation and testing for a unit root in the nonstationary autoregressive moving-average model. Journal of Time Series Analysis 16, 339-353. 